\title{
Cholesteric Liquid Crystal Inductive Asymmetric Polymerization: Synthesis of Chiral Polythiophene Derivatives from Achiral Monomers in a Cholesteric Liquid Crystal
}

\author{
Hiromasa Goto* \\ Institute of Materials Science, Tsukuba Research Center for \\ Interdisciplinary Materials Science (TIMS), \\ University of Tsukuba \\ Tsukuba, Ibaraki 305-8573, Japan \\ Correspondence to H. Goto, e-mail; gotoh@ims.tsukuba.ac.jp
}


4'-[(R)-1-Methyl-heptyloxy]biphenyl-4-carbonitrile $\quad[(R)-1] . \quad$ To a solution of 4-hydroxy-4-cyanobiphenyl (10.7 g, $55 \mathrm{mmol})$, DEAD (24.1 g, $55.3 \mathrm{mmol}$; in $40 \%$ toluene solution) in $60 \mathrm{~mL}$ of THF was added a solution of $(S)-(+)$-2-octanol (6.5 g, 50.2 $\mathrm{mmol})$, TPP (14.4 g, $55.1 \mathrm{mmol})$ in $30 \mathrm{~mL}$ of THF very slowly using pressure equalized dropping funnel. The resulting reaction mixture was stirred for a further $24 \mathrm{~h}$ under an argon atmosphere, TLC indicated completion of the reaction. The orange color reaction mixture was then evaporated to remove solvent. The solid was washed with water thoroughly and extracted with ether. Then the organic layer was evaporated to remove solvent. Purification by column chromatography (silica gel, $n$-hexane $/ \mathrm{CH}_{2} \mathrm{Cl}_{2}=1$ ) afforded $10.8 \mathrm{~g}$ (35.1 mmol) of a colorless liquid of $(R)-1$ in $70.0 \%$ yield. Anal. Calcd for $\mathrm{C}_{21} \mathrm{H}_{25} \mathrm{NO}_{4}$ : C, 82.04; H, 8.20, N, 4.56. Found: C, 82.20; H, 8.10, N, 4.55. IR ( KBr, cm $\left.{ }^{-1}\right)$ : $2930\left(v_{\mathrm{CH} 2}\right), 2255\left(v_{\mathrm{CN}}\right), 1603\left(v_{\mathrm{C}=\mathrm{C}}\right), 1248\left(v_{\mathrm{C}-\mathrm{O}-\mathrm{C}}\right), 823(\mathrm{CH}) .{ }^{1} \mathrm{H}$ NMR $(150 \mathrm{MHz}$, $\left.\mathrm{CDCl}_{3}, \mathrm{TMS}, \mathrm{ppm}\right): \delta=0.88\left(\mathrm{t}, J=7.08 \mathrm{~Hz}, 3 \mathrm{H}, \mathrm{CH}_{3}\right), 1.27-1.35\left(\mathrm{~m}, 6 \mathrm{H}, \mathrm{CH}_{2} \times 3\right), 1.46$ $\left(\mathrm{m}, 2 \mathrm{H}, \mathrm{CH}_{2} \mathrm{CH}_{3}\right), 1.60\left(\mathrm{~m}, 3 \mathrm{H}, \mathrm{C} * \mathrm{HCH}_{3} \mathrm{O}\right), 1.76\left(\mathrm{~m}, 2 \mathrm{H}, \mathrm{CH}_{2} \mathrm{CH}_{2} \mathrm{C}^{*} \mathrm{HCH}_{3} \mathrm{O}\right), 4.41$ (sextet, $\left.J=6.12 \mathrm{~Hz}, 1 \mathrm{H}, \mathrm{C}^{*} H \mathrm{CH}_{3} \mathrm{O}\right), 7.01(\mathrm{~d}, J=8.76 \mathrm{~Hz}, 2 \mathrm{H}, \mathrm{ph}), 7.51$ (d, $J=8.72 \mathrm{~Hz}$, $2 \mathrm{H}, \mathrm{Ph}), 7.63$ (d, $J=8.45 \mathrm{~Hz}, 2 \mathrm{H}, \mathrm{Ph}), 7.68(\mathrm{~d}, J=8.22 \mathrm{~Hz}, 2 \mathrm{H}, \mathrm{Ph}) .{ }^{13} \mathrm{C} \mathrm{NMR}(600 \mathrm{MHz}$, $\left.\mathrm{CDCl}_{3}, \mathrm{TMS}, \mathrm{ppm}\right): \delta=14.1\left(\mathrm{CH}_{3}\right), 19.7\left(\mathrm{C}^{*} \mathrm{HCH} \mathrm{H}_{3} \mathrm{O}\right), 22.6,25.5,29.3,31.8,36.4,74.0$ $\left(C^{*} \mathrm{HCH}_{3} \mathrm{O}\right), 110.0,116.2,119.1,127.0,128.3,131.1,132.5,145.3,158.9 .[\alpha]_{\mathrm{D}}{ }^{20}=+1.4$ $\circ\left(\mathrm{CHCl}_{3}\right)$.

4'-[(R)-1-Methyl-heptyloxy]biphenyl-4-carboxylic acid [(R)-2]. A solution of $(R)-1$ $(10.8 \mathrm{~g}, 35.1 \mathrm{mmol})$ in of ethanol $(120 \mathrm{~mL})$ was added to a solution of potassium hydroxide (49.4 g, $0.81 \mathrm{~mol})$ in water $(80 \mathrm{~mL})$ in an argon atmosphere. After one week, the reaction mixture was evaporated to remove solvent and filtration afforded white powder. The crude product was washed with large amount of $n$-hexane, dried in vacuo to yield white solid of $(R)-2$ (9.2 g, $28.2 \mathrm{mmol})$. Yield: $80 \%$. Anal. Calcd for $\mathrm{C}_{21} \mathrm{H}_{26} \mathrm{O}_{3}$ : C, 77.27; H, 8.03. Found: C, 77.20; H, 8.10. IR $\left(\mathrm{KBr}, \mathrm{cm}^{-1}\right): 2924\left(\mathrm{v}_{\mathrm{CH} 2}\right), 2668,2540,1680$ $\left(v_{\mathrm{C}=\mathrm{O}}\right), 1601\left(\mathrm{v}_{\mathrm{C}=\mathrm{C}}\right), 1283\left(\mathrm{v}_{\mathrm{C}-\mathrm{O}-\mathrm{C}}\right), 838\left(\delta_{\mathrm{CH}}\right) .{ }^{1} \mathrm{H}$ NMR $\left(600 \mathrm{MHz}, \mathrm{CDCl}_{3}, \mathrm{TMS}\right): \delta=0.88$ $\left(\mathrm{t}, J=6.96 \mathrm{~Hz}, 3 \mathrm{H}, \mathrm{CH}_{3}\right), 1.27-1.35\left(\mathrm{~m}, 6 \mathrm{H}, \mathrm{CH}_{2} \times 3\right), 1.47\left(\mathrm{~m}, 2 \mathrm{H}, \mathrm{CH}_{2} \mathrm{CH}_{3}\right), 1.60(\mathrm{~m}, 3 \mathrm{H}$, $\mathrm{C} * \mathrm{HCH}_{3} \mathrm{O}$ ), $1.77\left(\mathrm{~m}, 2 \mathrm{H}, \mathrm{CH}_{2} \mathrm{CH}_{2} \mathrm{C} * \mathrm{HCH}_{3} \mathrm{O}\right.$ ), 4.42 (sextet, $J=6.12 \mathrm{~Hz}, 1 \mathrm{H}, \mathrm{C}^{*} \mathrm{HCH}_{3} \mathrm{O}$ ), $6.98(\mathrm{~d}, J=8.76 \mathrm{~Hz}, 2 \mathrm{H}, \mathrm{Ph}), 7.57$ (d, $J=8.76 \mathrm{~Hz}, 2 \mathrm{H}, \mathrm{Ph}), 7.66$ (d, $J=8.46 \mathrm{~Hz}, 2 \mathrm{H}, \mathrm{Ph}$ ), $8.15(\mathrm{~d}, J=8.40 \mathrm{~Hz}, 2 \mathrm{H}, \mathrm{ph}) .{ }^{13} \mathrm{C} \mathrm{NMR}\left(150 \mathrm{MHz}, \mathrm{CDCl}_{3}, \mathrm{TMS}\right): \delta=14.1\left(\mathrm{CH}_{3}\right), 19.7$ $\left(\mathrm{C}^{*} \mathrm{HCH}_{3} \mathrm{O}\right), 22.6,25.5,29.3,31.8,36.5,74.0\left(C^{*} \mathrm{HCH}_{3} \mathrm{O}\right), 116.2,126.5,127.1,128.4$, 130.7, 131.9, 145.3, 158.9, $171.1(\mathrm{COOH})$. Liquid crystallinity: K160 (152) $\mathrm{S}_{\mathrm{X}} 166$ (159) SmC* 176 (173) $\mathrm{N}^{*} 195$ (192) I, in ${ }^{\circ} \mathrm{C}, \mathrm{K}$ : crystal, $\mathrm{S}_{\mathrm{X}}$ : unknown smectic phase, SmC*: chiral smectic $\mathrm{C}, \mathrm{N}^{*}$ chiral nematic, I: isotropic, blanket: cooling process. 
(+)-4-Methoxy-benzoic acid 4' -[(R)-1-methyl-heptyloxy]biphenyl-4-yl ester, $(R)$-3. A solution of $(R)-2(9.2 \mathrm{~g}, 28.2 \mathrm{mmol}), N-N^{\prime}$-dicyclohexylcarbodiimide (DCC) $(5.8 \mathrm{~g}$, $28.2 \mathrm{mmol}$ ) and 4-methyl aminopyridine (DMAP) (3.5 g, $28.2 \mathrm{mmol})$, and p-ethoxyphenol (3.8 g, $28.2 \mathrm{mmol}$ ) in $65 \mathrm{~mL}$ of $\mathrm{CH}_{2} \mathrm{Cl}_{2}$ was stirred for $24 \mathrm{~h}$ at room temperature. After evaporation of the solvent, purification of crude product by column chromatography (silica gel, $\mathrm{CH}_{2} \mathrm{Cl}_{2}$ ) followed by recrystallization from ethanol afforded $9.6 \mathrm{~g}(21.5 \mathrm{mmol})$ of white material. Yield $=76 \%$. Anal. Calcd for $\mathrm{C}_{29} \mathrm{H}_{34} \mathrm{O}_{4}: \mathrm{C}, 78.00 ; \mathrm{H}$, 7.67. Found: 78.00, H, 7.66. IR $\left(\mathrm{KBr}, \mathrm{cm}^{-1}\right): 2925\left(\mathrm{v}_{\mathrm{CH} 2}\right), 1726\left(\mathrm{v}_{\mathrm{C}=\mathrm{O}}\right), 1507\left(\mathrm{v}_{\mathrm{C}=\mathrm{C}}\right)$, $1250\left(\mathrm{v}_{\mathrm{C}-\mathrm{O}-\mathrm{C}}\right), 814\left(\delta_{\mathrm{CH}}\right) .{ }^{1} \mathrm{H} \mathrm{NMR}\left(\mathrm{CDCl}_{3}, 600 \mathrm{MHz}, \mathrm{TMS}, \mathrm{ppm}\right): \delta=0.88(\mathrm{t}, J=6.66$ $\left.\mathrm{Hz}, 3 \mathrm{H}, \mathrm{CH}_{3}\right), 1.27-1.36\left(\mathrm{~m}, 6 \mathrm{H}, \mathrm{CH}_{2} \times 3\right), 1.42\left(\mathrm{t}, J=6.96 \mathrm{~Hz}, 3 \mathrm{H}, \mathrm{phOCH}_{2} \mathrm{CH}_{3}\right), 1.46$ $\left(\mathrm{m}, 2 \mathrm{H}, \mathrm{CH}_{2} \mathrm{CH}_{3}\right), 1.59\left(\mathrm{~m}, 3 \mathrm{H}, \mathrm{C} * \mathrm{HCH} H_{3} \mathrm{O}\right), 1.77\left(\mathrm{~m}, 2 \mathrm{H}, \mathrm{CH}_{2} \mathrm{CH}_{2} \mathrm{C} * \mathrm{HCH}_{3} \mathrm{O}\right), 4.03$ (q, $J$ $=7.00 \mathrm{~Hz}, 2 \mathrm{H}$, ph-OCH $\left.\mathrm{CH}_{3}\right), 4.41$ (sextet, $\left.J=6.06 \mathrm{~Hz}, 1 \mathrm{H}, \mathrm{C}^{*} H \mathrm{CH}_{3} \mathrm{O}\right), 6.93(\mathrm{~d}, J=$ $8.94 \mathrm{~Hz}, 2 \mathrm{H}, \mathrm{Ph}), 6.98$ (d, $J=8.64 \mathrm{~Hz}, 2 \mathrm{H}, \mathrm{Ph}), 7.12$ (d, $J=8.88 \mathrm{~Hz}, 2 \mathrm{H}, \mathrm{Ph}), 7.57$ (d, $J=$ $8.58 \mathrm{~Hz}, 2 \mathrm{H}, \mathrm{Ph}), 7.67$ (d, J=8.28 Hz, 2H, Ph), 8.22 (d, J=8.22 Hz, 2H; Ph). ${ }^{13} \mathrm{C} \mathrm{NMR}$ $\left(\mathrm{CDCl}_{3}, 150 \mathrm{MHz}, \mathrm{TMS}, \mathrm{ppm}\right): \delta=14.1\left(\mathrm{CH}_{3}\right), 14.8\left(\mathrm{ph}-\mathrm{OCH}_{2} \mathrm{CH}_{3}\right), 19.7\left(\mathrm{C}^{*} \mathrm{HCH}_{3} \mathrm{O}\right)$, 22.6, 25.5, 29.3, 31.8, 36.5, $63.8\left(\mathrm{ph}-\mathrm{OCH}_{2} \mathrm{CH}_{3}\right), 74.0\left(C^{*} \mathrm{HCH}_{3} \mathrm{O}\right), 115.0,116.1,122.4$, 126.5, 127.5, 128.4, 130.7, 131.8, 144.3, 145.8, 156.6, 158.6, 165.5 (Ph-COO-Ph). Liquid crystallinity: K97 (72) $\mathrm{N}^{*} 135$ (133) I, in ${ }^{\circ} \mathrm{C}, \mathrm{K}$ : crystal, $\mathrm{N}^{*}$ : cholesteric, I: isotropic, blanket: cooling process. $[\alpha]_{\mathrm{D}}^{20}=+3.2^{\circ}\left(\mathrm{CHCl}_{3}\right)$.

2,2'-bis(trimethylstannyl)thiophene (4). A solution of thiophene (1 g, $12 \mathrm{mmol})$ and $N, N, N^{\prime}, N$ '-tetramethylethylenediamine (TMEDA, $2.9 \mathrm{~g}, 25 \mathrm{mmol}$ ) in $15 \mathrm{~mL}$ THF was stirred at $0{ }^{\circ} \mathrm{C}$. $n$-Butyl lithium in $n$-hexane $(16 \mathrm{~mL}, 24 \mathrm{mmol}$; in $1.5 \mathrm{M} n$-hexane solution) was added to this solution by pressure equalized dropping funnel for $10 \mathrm{~min}$. After completion of the addition, the mix solution was stirred for $1 \mathrm{~h}$ at $50{ }^{\circ} \mathrm{C}$. Subsequently, the solution was cooled to $0^{\circ} \mathrm{C}$ again, and trimethyltin chloride $(24 \mathrm{~mL}, 24 \mathrm{mmol}$; in $1.0 \mathrm{M}$ THF solution) was added and stirred for $24 \mathrm{~h}$. Saturated ammonium chloride/water solution was added to the mixture and washed several times, extracted by ether. The solution was dried over sodium sulfate, and evaporated. The pale yellow oil was recrystallized by hexane to afford $2 \mathrm{~g}$ of pale yellow needle crystal. Yield: $45 \%$. Anal. Calcd for $\mathrm{C}_{10} \mathrm{H}_{20} \mathrm{SSn}_{2}$ : C, 29.32; H, 4.92. Found: C,29.54, H, 5.05. IR (KBr, cm $\left.{ }^{-1}\right): 3054$, 2983, $2913\left(\mathrm{v}_{\mathrm{CH} 2, \mathrm{CH} 3}\right), 1476\left(\mathrm{v}_{\mathrm{C}=\mathrm{C}}\right), 797\left(\delta_{\mathrm{CH}}\right)$. NMR $\left(\mathrm{CDCl}_{3}, 600 \mathrm{MHz}, \mathrm{TMS}, \mathrm{ppm}\right): \delta=$ 0.30 (s, $\left.18 \mathrm{H}, \mathrm{CH}_{3}\right), 7.31$ (s, $\left.2 \mathrm{H}, \mathrm{ThH}\right) .{ }^{13} \mathrm{C} \mathrm{NMR}\left(\mathrm{CDCl}_{3}, 150 \mathrm{MHz}, \mathrm{TMS}, \mathrm{ppm}\right): \delta=-7.2$, 136.8, 144.0. 
5,5'-Bis-trimethylstannanyl-2,2';5',2''-terthiophene (6). was prepared using the same

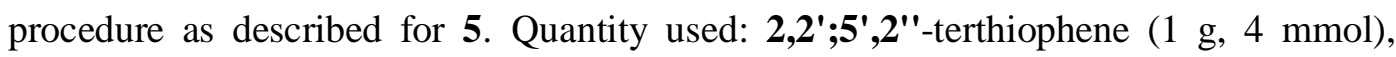
TMEDA (1.21 g, $10.4 \mathrm{mmol}), n$-butyl lithium (3.44 mL, $50 \mathrm{mmol} ; 2.76 \mathrm{M}$ in $n$-hexane), trimethyltin chloride $(8.98 \mathrm{~mL}, 8.98 \mathrm{mmol}$; in $1.0 \mathrm{M}$ THF solution). $\mathrm{Y}=1.33 \mathrm{~g}, 2.31$ mmol (57 \%). Anal. Calcd for $\mathrm{C}_{18} \mathrm{H}_{24} \mathrm{~S}_{3} \mathrm{Sn}_{2}$ : C, 37.67; H, 4.51. Found: C, 38.05; H,4.27. ${ }^{1} \mathrm{H} \mathrm{NMR}\left(\mathrm{CDCl}_{3}, 270 \mathrm{MHz}, \mathrm{TMS}, \mathrm{ppm}\right): \delta=0.38\left(\mathrm{~s}, 18 \mathrm{H}, \mathrm{CH}_{3}\right), 7.08(\mathrm{~d}, J=3.4 \mathrm{~Hz}, 4 \mathrm{H}$, $\mathrm{Th}), 7.26$ (d, $J=3.4 \mathrm{~Hz}, 2 \mathrm{H}$, Th).

2,5-Dibromo-benzoic acid undecyl ester (7). A solution of 2,5-dibromobenzoic acid $(10 \mathrm{~g}, 36 \mathrm{mmol})$, and DEAD (15.7 g, $40 \mathrm{wt} \%$ in toluene;36 mol) in $20 \mathrm{~mL}$ of THF was added dropwise with pressure dropping funnel to a stirred mixture of 1-undecanol (6.6 g, $36 \mathrm{mmol})$, and TPP $(9.4 \mathrm{~g}, 36 \mathrm{mmol})$ in THF $(20 \mathrm{~mL})$. The resulting reaction mixture was stirred for a further $24 \mathrm{~h}$ under an argon atmosphere, TLC indicated completion of the reaction. The orange color reaction mixture was then evaporated to remove solvent. The solid was washed with water thoroughly and extracted with ether. Then the organic layer was evaporated to remove solvent. Purification by column chromatography (silica gel, $\mathrm{CH}_{2} \mathrm{Cl}_{2}$ ) followed by recrystallization form ethanol afforded $7.4 \mathrm{~g}(17 \mathrm{mmol})$ of a white crystal of 7 in $47.0 \%$ yield. Anal. Calcd for $\mathrm{C}_{18} \mathrm{H}_{26} \mathrm{Br}_{2} \mathrm{O}_{2}: \mathrm{C}, 49.79 ; \mathrm{H}, 6.04$. Found: $\mathrm{C}$, 49.91; H, 5.85. IR $\left(\mathrm{cm}^{-1}\right): 2935,2856\left(\mathrm{v}_{\mathrm{CH} 2}, \mathrm{CH} 3\right), 1701\left(v_{\mathrm{C}=\mathrm{O}}\right), 1270\left(\mathrm{v}_{\mathrm{C}-\mathrm{O}-\mathrm{C}}\right) .{ }^{1} \mathrm{H}$ NMR $\left(\mathrm{CDCl}_{3}, 600 \mathrm{MHz}, \mathrm{TMS}, \mathrm{ppm}\right): \delta=0.38\left(\mathrm{~s}, J=6.8 \mathrm{~Hz}, 3 \mathrm{H}, \mathrm{CH}_{3}\right), 1.24-1.27(\mathrm{~m}, 14 \mathrm{H}$, $\mathrm{CH}_{2}$ ), 1.33 (quint, $J=7.2 \mathrm{~Hz}, 2 \mathrm{H}, \mathrm{OCH}_{2} \mathrm{CH}_{2} \mathrm{CH}_{2}$ ), 1.67 (quint, $J=6.7 \mathrm{~Hz}, 2 \mathrm{H}$, $\left.\mathrm{OCH}_{2} \mathrm{CH}_{2}\right), 4.24\left(\mathrm{t}, J=6.7 \mathrm{~Hz}, 2 \mathrm{H}, \mathrm{OCH}_{2}\right), 7.32(\mathrm{~d}, J=6.1 \mathrm{~Hz}, 1 \mathrm{H}, \mathrm{Ph}), 7.41(\mathrm{~d}, J=8.5$ $\mathrm{Hz}, 1 \mathrm{H}, \mathrm{Ph}), 7.80$ (s, 1H; Ph). ${ }^{13} \mathrm{C}$ NMR $\left(\mathrm{CDCl}_{3}, 150 \mathrm{MHz}, \mathrm{TMS}, \mathrm{ppm}\right): \delta=14.5$ $\left(\mathrm{CH}_{2} \mathrm{CH}_{3}\right), 23.1,26.4,29.0,29.6,29.8,29.9,30.0,30.1,32.3,66.6,120.7,121.4,134.5$, $135.7,136.1,165.3$. 


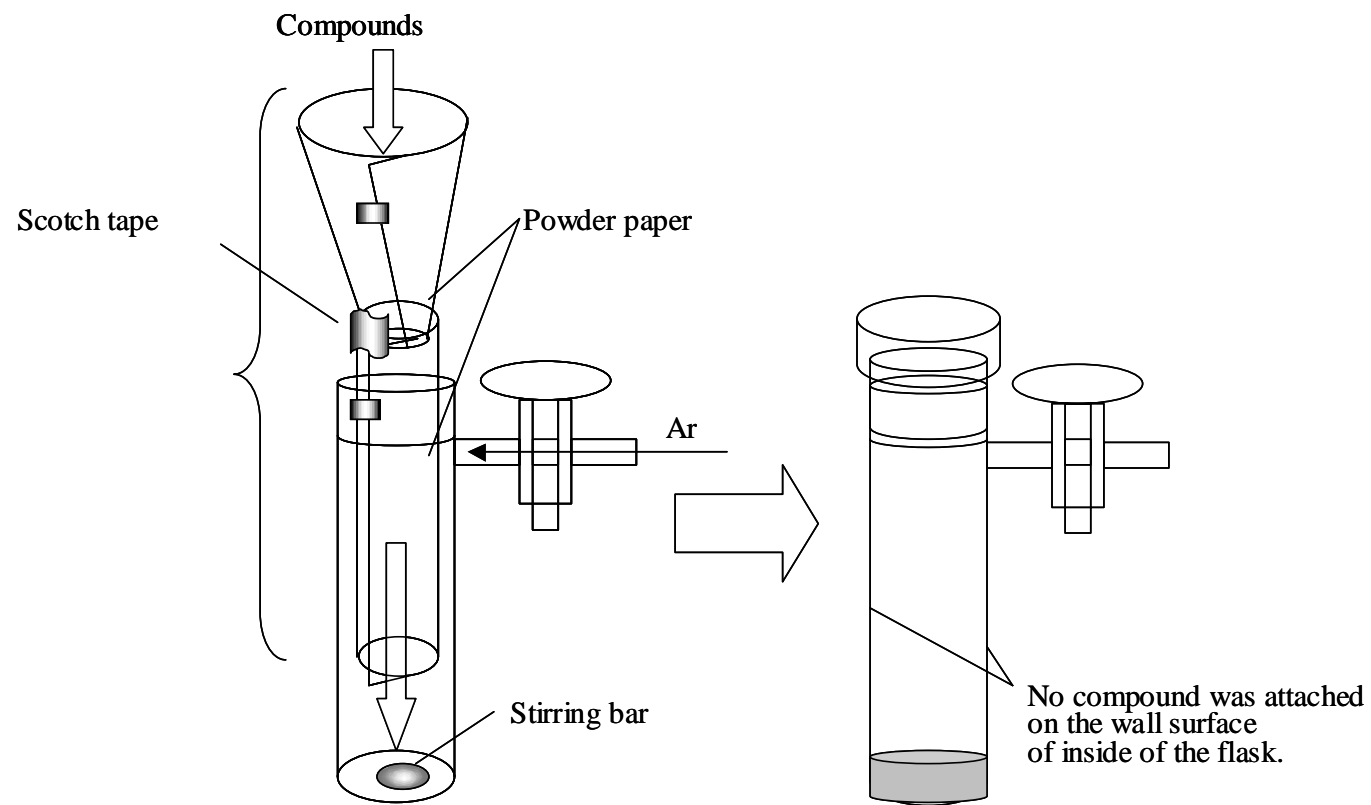

Figure S1. Schematic illustration of custom-made paper funnel for transferring cholesteric liquid crystal compound (powder form at room temperature) to the reaction flask. 